\title{
Compact Electro-Thermal Models of Interconnects
}

Lorenzo Codecasa*1

\author{
${ }^{1}$ Politecnico di Milano, Dipartimento di Elettronica, Informazione e Bioingegneria, Milan, Italy \\ * Corresponding Author: Codecasa@elet.polimi.it,+39 0223993534
}

Abstract

In this paper a novel approach is proposed for generating nonlinear compact models of the dynamic electrothermal problems modeling interconnects. The method is very efficient and leads to accurate approximations of the space-time distribution of voltages, currents and temperature rises within the interconnects for all waveforms of the port variables.

\section{Introduction}

According to the predictions of the International Technology Roadmap for Semiconductors [1], the realization of efficient interconnects is a challenging problem along the route towards nanoelectronics, since they are required to satisfy stringent requirements in terms of electrical and thermal behaviors. In fact the scaling of very-large-scale integration structures implies increasing current densities that result in greater Joule heating and in greater temperature rises. These thermal effects introduce a strong degradation of the electrical speed performance.

In the last decades large efforts have been devoted in literature for modeling the electrical behavior of interconnects, mainly resorting to equivalent electric transmission lines. In order to alleviate the computational burden in the simulation at circuit level, many techniques have been proposed for modeling transmission lines by means of models of reduced complexity, known as compact models [2].

However in order to properly take into account temperature effects on the electrical performance of interconnects, the electrical models of interconnects cannot be used as they are, but have to be complicated for modeling electro-thermal effects. The premier choice is an electro-thermal model composed by a transmission line, modeling the electric effect, coupled to a transmission line, modeling the thermal effect [3]-[28]. Unfortunately, as a consequence of the nonlinearity of such model, the results reported in literature for generating compact models of electric transmission lines cannot be applied. A novel approach for generating compact models is thus both crucial and challenging.

In this paper an approach for generating compact models of coupled electro-thermal transmission lines is proposed. It takes into account the dependences of electric resistivity on temperature relevant for interconnects. A projection is applied in such a way that the nonlinear structure of the electro-thermal equations is preserved. The projection space is obtained from the Volterra series expansion [29] of the solution of the electro-thermal problem.

The details of the derivation are provided for the simplest case of a one-conductor electric transmission line coupled to a one-conductor thermal transmission line. The investigation af a simple example problem shows that the proposed approach is efficient and leads to compact and accurate electro-thermal models, ready to be used in simulations at the circuit level, for temperature rises well above those needed for interconnect analysis.

\section{Formulation of the electro-thermal problem}

The equation of an electric transmission line, here assumed to be of RC type, can be written in the form

$$
\begin{aligned}
& c(x) \frac{\partial v}{\partial t}(x, t)+\frac{\partial i}{\partial x}(x, t)=0 \\
& \frac{\partial v}{\partial x}(x, t)+r(x, u(x, t)) i(x, t)=0
\end{aligned}
$$

in which $v(x, t)$ and $i(x, t)$ are the voltage and current at position $0 \leqslant x \leqslant L$ and at time instant $t$. The electric capacitance per unit of length is $c(x)$ and the electric resistance per unit of length is $r(x, u(x, t))$, assumed to be dependent on the temperature rise $u(x, t)$ in the common form

$$
r(x, u(x, t))=r(x, 0)(1+\mu(x) u(x, t)),
$$

which is very accurate for metals in interconnects. For the sake of simplicity, boundary conditions are assumed of the form

$$
\begin{aligned}
& i(0, t)=I(t), \\
& v(L, t)=0
\end{aligned}
$$

in which $I(t)$ is the port electric current. Initial conditions are

$$
v(x, 0)=0 .
$$

The temperature rise distribution $u(x, t)$ is modeled by a thermal transmission line ruled by equations

$$
\begin{aligned}
m(x) \frac{\partial u}{\partial t}(x, t)+\frac{\partial}{\partial x}\left(-k_{t}(x) \frac{\partial u}{\partial x}(x, t)\right)+ & \\
+ & k_{n}(x) u(x, t)=g(x, t),
\end{aligned}
$$

in which the thermal capacitance per unit of length is $m(x)$, the thermal conductances per unit of length are $k_{t}(x)$ and $k_{n}(t)$. The power density $g(x, t)$ is due to the Joule heating 
in the electric transmission line and can be written in the form

$$
g(x, t)=-\frac{\partial v}{\partial x}(x, t) i(x, t) .
$$

The equations of the thermal transmission line is completed by boundary conditions, of Robin's type, in the form

$$
\begin{aligned}
& -k_{t}(0) \frac{\partial u}{\partial x}(0, t)=h(0) u(0, t) \\
& k_{t}(L) \frac{\partial u}{\partial x}(L, t)=h(L) u(L, t)
\end{aligned}
$$

and by initial conditions

$$
u(x, 0)=0 .
$$

The port variable $I(t)$ of the electric transmission line is completed by introducing the port voltage

$$
V(t)=v(0, t) .
$$

Previous equations of the coupled electric and thermal transmission lines provide a well-known electro-thermal model of interconnects [25]. These equations are here elaborated for constructing a nonlinear compact electrothermal model. To this aim a novel projection approach is used, which preserves the nonlinear structure of the electrothermal equations. Thus, $v(x, t)$ is approximated in the form

$$
v(x, t)=\sum_{i=1}^{\hat{m}_{v}} v_{i}(x) \hat{v}_{i}(t)
$$

in which $v_{i}(x)$, with $i=1, \ldots, \hat{m}_{v}$, are a small number of basis functions, to be determined. For the sake of robustness they are assumed to form an orthonormal basis such that

$$
\int_{0}^{L} c(x) v_{i}(x) v_{j}(x) d x=\delta_{i j}, \quad i, j=1, \ldots, \hat{m}_{v} .
$$

Similarly $i(x, t)$ is approximated in the form

$$
i(x, t)=\sum_{j=1}^{\hat{m}_{i}} i_{j}(x) \hat{\imath}_{j}(t)
$$

in which $i_{j}(x)$, with $j=1, \ldots, \hat{m}_{i}$, are a small number of basis functions, to be determined. For the sake of robustness they are assumed to form an orthonormal basis such that

$$
\int_{0}^{L} i_{i}(x) i_{j}(x) d x=\delta_{i j}, \quad i, j=1, \ldots, \hat{m}_{i} .
$$

Lastly $u(x, t)$ is approximated in the form

$$
u(x, t)=\sum_{k=1}^{\hat{m}_{u}} u_{k}(x) \hat{u}_{k}(t)
$$

in which $u_{j}(x)$, with $j=1, \ldots, \hat{m}_{u}$, are a small number of basis functions, to be determined. Again, for the sake of robustness they are assumed to form an orthonormal basis such that

$$
\int_{0}^{L} m(x) u_{i}(x) u_{j}(x) d x=\delta_{i j}, \quad i, j=1, \ldots, \hat{m}_{u} .
$$

With these assumptions, multiplying (1) by $u_{i}(x)$, integrating with respect to $x$ in $[0, L]$, integrating by parts and recalling (4), (5) it results in

$$
\frac{d \hat{v}_{i}}{d t}(t)+\sum_{j=1}^{\hat{m}_{i}} \hat{g}_{i j}^{1} \hat{\imath}_{j}(t)=v_{i}(0) I(t),
$$

in which

$$
\hat{g}_{i j}^{1}=-\int_{0}^{L} \frac{d v_{i}}{d x}(x) i_{j}(x) d x
$$

Also, multiplying (2) by $i_{j}(x)$, recalling (3) and integrating with respect to $x$ in $[0, L]$, it results in

$$
-\sum_{j=1}^{\hat{m}_{v}} \hat{g}_{j i}^{1} \hat{v}_{j}(t)+\sum_{j=1}^{\hat{m}_{i}} \hat{r}_{i j}^{1} \hat{\imath}_{j}(t)+\sum_{j=1}^{\hat{m}_{i}} \sum_{k=1}^{\hat{m}_{u}} \hat{r}_{i j k}^{2} \hat{\imath}_{j}(t) \hat{u}_{k}(t)=0,
$$

in which

$$
\begin{aligned}
& \hat{r}_{i j}^{1}=\int_{0}^{L} r(x, 0) i_{i}(x) i_{j}(x) d x \\
& \hat{r}_{i j k}^{2}=\int_{0}^{L} \mu(x) r(x, 0) i_{i}(x) i_{j}(x) u_{k}(x) d x .
\end{aligned}
$$

In a similar way, multiplying (7) by $u_{j}(x)$, integrating with respect to $x$ in $[0, L]$, integrating by parts and recalling $(8),(9)$ and (10) it results in

$$
\frac{d \hat{u}_{i}}{d t}(t)+\sum_{j=1}^{\hat{m}_{u}} \hat{k}_{i j} \hat{u}_{j}(t)=\sum_{j=1}^{\hat{m}_{v}} \sum_{k=1}^{\hat{m}_{i}} \hat{g}_{i j k}^{2} \hat{v}_{j}(t) \hat{\imath}_{k}(t),
$$

in which

$$
\begin{gathered}
\hat{k}_{i j}=\int_{0}^{L}\left(k_{t}(x) \frac{d u_{i}}{d x}(x) \frac{d u_{j}}{d x}(x)+k_{n}(x) u_{i}(x) u_{j}(x)\right) d x+ \\
+h(0) u_{i}(0) u_{j}(0)+h(L) u_{i}(L) u_{j}(L), \\
\hat{g}_{i j k}^{2}=-\int_{0}^{L} u_{i}(x) \frac{d v_{j}}{d x}(x) i_{k}(x) d x .
\end{gathered}
$$

From (5), (11), initial conditions for the compact model follow

$$
\begin{aligned}
& \hat{v}_{i}(t)=0, \quad i=1, \ldots, \hat{m}_{v} \\
& \hat{u}_{i}(t)=0, \quad i=1, \ldots, \hat{m}_{u} .
\end{aligned}
$$

The compact thermal model provides an approximation of the port voltage given by

$$
\hat{V}(t)=\sum_{j=1}^{\hat{m}_{v}} v_{j}(0) \hat{v}_{j}(t)
$$

It also provides an approximation of the whole voltage, current and temperature rise spatio-temporal distributions in 
the forms

$$
\begin{aligned}
& \hat{v}(x, t)=\sum_{j=1}^{\hat{m}_{v}} v_{j}(x) \hat{v}_{j}(t), \\
& \hat{\imath}(x, t)=\sum_{j=1}^{\hat{m}_{i}} i_{j}(x) \hat{\imath}_{j}(t), \\
& \hat{u}(x, t)=\sum_{j=1}^{\hat{m}_{u}} u_{j}(x) \hat{u}_{j}(t) .
\end{aligned}
$$

\section{Volterra series moments}

It is well known that the output $y(t)$ of a SISO timeinvariant nonlinear dynamical system of input $u(t)$, under proper regularity assumptions, can be given a Volterra series expansion [28], [29]. Such Volterra series expansion is now computed for the variables in the nonlinear electro-thermal transmission line equations. To this aim, firstly (2), (3) are rewritten in the form

$$
\frac{\partial v}{\partial x}(x, t)+r(x, 0)(1+\mu(x) u(x, t)) i(x, t)=0
$$

and (4), (7) are rewritten in the form

$$
\begin{aligned}
m(x) \frac{\partial u}{\partial t}(x, t)+ & \frac{\partial}{\partial x}\left(-k_{t}(x) \frac{\partial u}{\partial x}(x, t)\right)+ \\
& +k_{n}(x) u(x, t)=-\frac{\partial v}{\partial x}(x, t) i(x, t),
\end{aligned}
$$

Then Volterra series terms $V_{m}\left(x, \mathbf{s}_{m}\right), I_{m}\left(x, \mathbf{s}_{m}\right), U\left(x, \mathbf{s}_{m}\right)$, respectively for $v(x, t), i(x, t), u(x, t)$ are computed, in which the notation $\mathbf{s}_{m}=\left(s_{1}, s_{2}, \ldots, s_{m}\right)$ is adopted. Using the properties of the theory of Volterra series expansions, it results in

$$
\begin{gathered}
\left(\sum_{k=1}^{m} s_{k}\right) c(x) V_{m}\left(x, \mathbf{s}_{m}\right)+\frac{d I_{m}}{d x}\left(x, \mathbf{s}_{m}\right)=0, \\
\frac{d V_{m}}{d x}\left(x, \mathbf{s}_{m}\right)+r(x, 0) I_{m}\left(x, \mathbf{s}_{m}\right)+\mu(x) r(x, 0) \cdot \\
\cdot \sum_{k=1}^{m-1} \sum_{\left|\mathbf{p}_{m}\right|=k} U_{k}\left(x, \mathbf{s}_{\mathbf{p}_{m}}\right) I_{m-k}\left(x, \mathbf{s}_{\mathbf{p}_{m}^{\prime}}\right) /\left(\begin{array}{c}
m \\
k
\end{array}\right)=0,
\end{gathered}
$$

with boundary conditions

$$
\begin{aligned}
& I_{m}\left(0, \mathbf{s}_{m}\right)=\delta_{m 1}, \\
& V_{m}\left(L, \mathbf{s}_{m}\right)=0,
\end{aligned}
$$

and in

$$
\begin{aligned}
& \left(\sum_{k=1}^{m} s_{k}\right) m(x) U_{m}\left(x, \mathbf{s}_{m}\right)+ \\
& +\frac{d}{d x}\left(-k_{t}(x) \frac{d U_{m}}{d x}\left(x, \mathbf{s}_{m}\right)\right)+k_{n}(x) U_{m}\left(x, \mathbf{s}_{m}\right)= \\
& =-\sum_{k=1}^{m-1} \sum_{\left|\mathbf{p}_{m}\right|=k} \frac{d V_{k}}{d x}\left(x, \mathbf{s}_{\mathbf{p}_{m}}\right) I_{m-k}\left(x, \mathbf{s}_{\mathbf{p}_{m}^{\prime}}\right) /\left(\begin{array}{c}
m \\
k
\end{array}\right),
\end{aligned}
$$

with boundary conditions

$$
\begin{aligned}
& -k_{t}(0) \frac{d U_{m}}{d x}\left(0, \mathbf{s}_{m}\right)=h(0) U_{m}\left(0, \mathbf{s}_{m}\right) \\
& \left.+k_{t}(L) \frac{d U_{m}}{d x}\left(L, \mathbf{s}_{m}\right)=h(L) U_{m}\left(L, \mathbf{s}_{m}\right)\right) .
\end{aligned}
$$

In these expressions $\mathbf{p}_{m}$ is a $m$-vector of zeros and ones, and $\mathbf{p}_{m}^{\prime}$ is the vector obtained from $\mathbf{p}_{m}$ by exchanging all zeros with ones and ones with zeros. Vector $\mathbf{s}_{\mathbf{p}_{m}}$ is a vector obtained by selecting the elements of $\mathbf{s}_{m}$ corresponding to the ones of $\mathbf{p}_{m}$.

Around a chosen value $\boldsymbol{\sigma}_{m}$ of $\mathbf{s}_{m}$ Taylor's series expansions of $V_{m}\left(x, \mathbf{s}_{m}\right), I_{m}\left(x, \mathbf{s}_{m}\right), U_{m}\left(x, \mathbf{s}_{m}\right)$ can be written in terms of their moments in the form

$$
\begin{aligned}
& V_{m}\left(x, \mathbf{s}_{m}\right)=\sum_{\boldsymbol{\alpha}_{m}} V_{m, \boldsymbol{\alpha}_{m}}\left(x, \boldsymbol{\sigma}_{m}\right)\left(\mathbf{s}_{m}-\boldsymbol{\sigma}_{m}\right)^{\boldsymbol{\alpha}_{m}}, \\
& I_{m}\left(x, \mathbf{s}_{m}\right)=\sum_{\boldsymbol{\alpha}_{m}} I_{m, \boldsymbol{\alpha}_{m}}\left(x, \boldsymbol{\sigma}_{m}\right)\left(\mathbf{s}_{m}-\boldsymbol{\sigma}_{m}\right)^{\boldsymbol{\alpha}_{m}}, \\
& U_{m}\left(x, \mathbf{s}_{m}\right)=\sum_{\boldsymbol{\alpha}_{m}} I_{m, \boldsymbol{\alpha}_{m}}\left(x, \boldsymbol{\sigma}_{m}\right)\left(\mathbf{s}_{m}-\boldsymbol{\sigma}_{m}\right)^{\boldsymbol{\alpha}_{m}},
\end{aligned}
$$

in which $\boldsymbol{\alpha}_{m}$ are multi-indices of $m$ elements. Using these expressions in previous equations it results in

$$
\begin{aligned}
& \left(\sum_{k=1}^{m} \sigma_{k}\right) c(x) V_{m, \boldsymbol{\alpha}_{m}}\left(x, \boldsymbol{\sigma}_{m}\right)+\frac{d I_{m, \boldsymbol{\alpha}_{m}}}{d x}\left(x, \boldsymbol{\sigma}_{m}\right)=0, \\
& \frac{d V_{m, \boldsymbol{\alpha}_{m}}}{d x}\left(x, \boldsymbol{\sigma}_{m}\right)+r(x, 0) I_{m, \boldsymbol{\alpha}_{m}}\left(x, \boldsymbol{\sigma}_{m}\right)+\mu(x) r(x, 0) \cdot \\
& \cdot \sum_{k=1}^{m-1} \sum_{\left|\mathbf{p}_{m}\right|=k} U_{k, \boldsymbol{\alpha}_{\mathbf{p}_{m}}}\left(x, \boldsymbol{\sigma}_{\mathbf{p}_{m}}\right) I_{m-k, \boldsymbol{\alpha}_{\mathbf{p}_{m}^{\prime}}}\left(x, \boldsymbol{\sigma}_{\mathbf{p}_{m}^{\prime}}\right) /\left(\begin{array}{c}
m \\
k
\end{array}\right)=0,
\end{aligned}
$$

with boundary conditions

$$
\begin{aligned}
& I_{m, \boldsymbol{\alpha}_{m}}\left(0, \boldsymbol{\sigma}_{m}\right)=\delta_{m 1} \delta_{\boldsymbol{\alpha}_{m} \mathbf{0}} \\
& V_{m, \boldsymbol{\alpha}_{m}}\left(L, \boldsymbol{\sigma}_{m}\right)=0
\end{aligned}
$$

and in

$$
\begin{aligned}
& \left(\sum_{k=1}^{m} \sigma_{k}\right) m(x) U_{m, \boldsymbol{\alpha}_{m}}\left(x, \boldsymbol{\sigma}_{m}\right)+ \\
& +\frac{d}{d x}\left(-k_{t}(x) \frac{d U_{m, \boldsymbol{\alpha}_{m}}}{d x}\left(x, \boldsymbol{\sigma}_{m}\right)\right)+k_{n}(x) U_{m, \boldsymbol{\alpha}_{m}}\left(x, \boldsymbol{\sigma}_{m}\right)= \\
& =-\sum_{k=1}^{m-1} \sum_{\left|\mathbf{p}_{m}\right|=k} \frac{d V_{k, \boldsymbol{\alpha}_{\mathbf{p}_{m}}}}{d x}\left(x, \boldsymbol{\sigma}_{\mathbf{p}_{m}}\right) I_{m-k, \boldsymbol{\alpha}_{\mathbf{p}_{m}^{\prime}}}\left(x, \boldsymbol{\sigma}_{\mathbf{p}_{m}^{\prime}}\right) /\left(\begin{array}{c}
m \\
k
\end{array}\right),
\end{aligned}
$$

with boundary conditions

$$
\begin{aligned}
& -k_{t}(0) \frac{d U_{m, \boldsymbol{\alpha}_{m}}}{d x}\left(0, \boldsymbol{\sigma}_{m}\right)=h(0) U_{m, \boldsymbol{\alpha}_{m}}\left(0, \boldsymbol{\sigma}_{m}\right), \\
& +k_{t}(L) \frac{d U_{m, \boldsymbol{\alpha}_{m}}}{d x}\left(L, \boldsymbol{\sigma}_{m}\right)=h(L) U_{m, \boldsymbol{\alpha}_{m}}\left(L, \boldsymbol{\sigma}_{m}\right) .
\end{aligned}
$$

Thus the first moments $V_{m, \boldsymbol{\alpha}_{m}}\left(x, \boldsymbol{\sigma}_{m}\right), I_{m, \boldsymbol{\alpha}_{m}}\left(x, \boldsymbol{\sigma}_{m}\right)$, $U_{m, \boldsymbol{\alpha}_{m}}\left(x, \boldsymbol{\sigma}_{m}\right)$ around a chosen expansion point $\boldsymbol{\sigma}_{m}$ with $\left|\boldsymbol{\alpha}_{m}\right| \leqslant q_{m}$ can be iteratively determined at the cost of the solution of $\left(\begin{array}{c}q_{m}+m \\ q_{m}\end{array}\right)$ linear electric transmission lines equations and linear thermal transmission lines equations, in 
the frequency domain for the real values $\sigma_{1}+\sigma_{2}+\cdots+\sigma_{m}$ of complex frequency.

However, from all previous equations it is noted that $V_{m}\left(x, \mathbf{s}_{m}\right)=I_{m}\left(x, \mathbf{s}_{m}\right)=0$ for $m$ even and $U_{m}\left(x, \mathbf{s}_{m}\right)=$ 0 for $m$ odd. Thus $V_{m, \boldsymbol{\alpha}_{m}}\left(x, \boldsymbol{\sigma}_{m}\right)=I_{m, \boldsymbol{\alpha}_{m}}\left(x, \boldsymbol{\sigma}_{m}\right)=0$ for $m$ even and all values of $\boldsymbol{\alpha}_{m}$ and $U_{m, \boldsymbol{\alpha}_{m}}\left(x, \boldsymbol{\sigma}_{m}\right)=0$ for $m$ odd and all values of $\boldsymbol{\alpha}_{m}$. All these moments have not to be computed explicitly.

The values of the moments of $V_{m, \boldsymbol{\alpha}_{m}}\left(x, \boldsymbol{\sigma}_{m}\right)$, $I_{m, \boldsymbol{\alpha}_{m}}\left(x, \boldsymbol{\sigma}_{m}\right), U_{m, \boldsymbol{\alpha}_{m}}\left(x, \boldsymbol{\sigma}_{m}\right)$, for some choices of the expansion point $\boldsymbol{\sigma}_{m}$, and expansion order $q_{m}$, with $m=$ $1, \ldots, r$, are then used for determining, by orthonormalization, the basis functions $v_{i}(x)$, with $i=1, \ldots, \hat{m}_{v}, i_{i}(x)$, with $i=1, \ldots, \hat{m}_{i}$ and $u_{i}(x)$, with $i=1, \ldots, \hat{m}_{u}$ and for the generation of the nonlinear electro-thermal compact model. As it can be proven, this ensures that the chosen moments in the Volterra series expansion of $v(x, t), i(x, t)$, $u(x, t)$ and $T(t)$ are matched by the corresponding moments in the Volterra series expansion of their approximations $\hat{v}(x, t), \hat{\imath}(x, t), \hat{u}(x, t)$ and $\hat{V}(t)$. Moreover additional moments of $V(t)$ with respect to the chosen ones are matched by $\hat{V}(t)$. The details are not reported here.

The proposed approach has been here discussed in terms of the continuous electro-thermal transmission line equations. It can however be straightforwardly reformulated in terms of any lumped circuit approximation of such transmission lines. The proposed approach is applied in the next section to such a lumped circuit approximation. In this case it is shown that the method is efficient. In fact the determination of the compact electro-thermal model, does not require the demanding solution of the nonlinear circuit in the time domain, but only the solution of few linear circuits in the complex frequency domain, which is less computationally expensive. The method is accurate. In fact the error with respect to the exact spatio-temporal distributions of voltages, currents and temperature rises rapidly tends to zero increasing $\hat{m}$. This is not only the consequence of the choice of the basis functions used for projection but also of the particular projection method adopted which preserves the non-linear structure of the electro-thermal problem. As a consequence of the structure preserving projection, the method is also robust. In fact no instabilities of the nonlinear compact models have been observed.

\section{Numerical Application}

Normalized material data have been assumed. The transmission line of length $L=1$ is divided into two parts of equal length: in the first part, $r(x)=1, \mu(x)=0.5$, $c(x)=0.1, k_{t}(x)=k_{n}(x)=1, m(x)=1, h(0)=0$; in the second part, $r(x)=5, \mu(x)=0, c(x)=0.1, k_{t}(x)=1$, $k_{n}(x)=0.5, m(x)=1, h(1)=\infty$.

A lumped circuit approximating of this problem having 20,000 nodes is introduced. A nonlinear dynamical compact electro-thermal model is generated from such lumped circuit using the proposed approach. The chosen values of the expansion points $\boldsymbol{\sigma}_{m}$ and of the computed moments $\boldsymbol{\alpha}_{m}$ are reported below.

\begin{tabular}{|l|l|l|}
\hline$m$ & $\boldsymbol{\sigma}_{m}$ & $\boldsymbol{\alpha}_{m}$ \\
\hline 1 & $10^{-2}$ & 0 \\
& & 1 \\
& & 2 \\
& & 3 \\
& $10^{0}$ & 0 \\
& & 1 \\
\hline 2 & $\left(10^{-2}, 10^{-2}\right)$ & $(0,0)$ \\
& & $(1,0)$ \\
& & $(1,1)$ \\
& & $(2,0)$ \\
& $\left(10^{0}, 10^{-2}\right)$ & $(0,0)$ \\
& & $(1,0)$ \\
\hline 3 & $\left(10^{-2}, 10^{-2}, 10^{-2}\right)$ & $(0,0,0)$ \\
\hline 4 & $\left(10^{-2}, 10^{-2}, 10^{-2}, 10^{-2}\right)$ & $(0,0,0,0)$ \\
\hline
\end{tabular}

The construction of the compact electro-thermal models requires the solution of 7 linear electric problems and 7 thermal problems at frequencies on the real axis. The solution of all these symmetric positive definite systems of equations, by means of the conjugate gradient algorithm with incomplete Cholesky preconditioning, requires about 1 second. After orthonormalization of the determined moments, a compact model with $\hat{m}_{v}=\hat{m}_{i}=7$ and $\hat{m}_{u}=7$ is generated. The nonlinear compact thermal model is represented by a DAE of index 1 . Thus any stiff numerical solver can be used for its solution. Each simulation in the time domain, shown in Figs. $1-6$, requires less than 1 second using ode $15 \mathrm{~s}$ in Matlab, on a 2.3 Ghz Intel Core i7. Instead the solution in the time domain of the lumped circuit modeling the interconnect required about 5 minutes for each simulation.

The accuracy of the compact model is rather insensitive to the exact choice of the expansion points or matching orders. The method is very accurate for all waveforms of the current $I(t)$, as outlined in Figs 4-6, in which also the solution of the linear problem, for $\mu(x)=0$, is reported. The limit of accuracy of the generated compact model is reached when the temperature rise $u$ reaches the value 100 . At this value, since $\mu(x)=0.5$, the electric resistivity is increased by a factor of 50 which is far more than what is required in the analysis of interconnects [1].

\section{Conclusions}

In this paper a novel approach has been proposed for generating nonlinear compact electro-thermal models of transmission lines. The approach exhibits high levels of accuracy for very small state space dimensions of the model. It is also very efficient since it requires the solution of few linear electric and thermal problems in the frequency domain. Such compact models can be used to accurately approximating not only the port variables of the interconnects but also the whole spatio-temporal distribution of voltage, current and temperature rise within the electric and thermal transmission lines modeling an interconnect.

\section{REFERENCES}

[1] International Technology Roadmap for Semiconductors, 2011, http://public.itrs.net. 


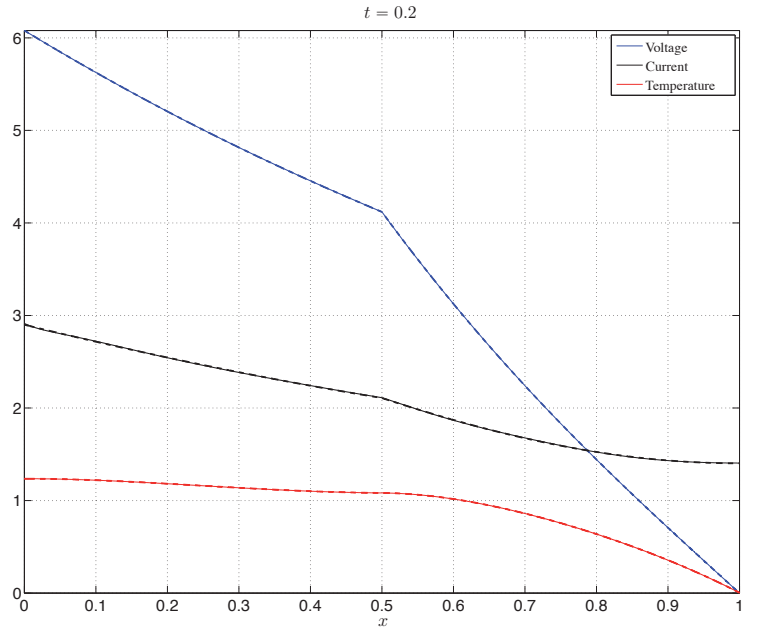

Fig. 1. Spacial distribution of voltage, current and temperature rise due to a current $I(t)$ constituted by a unit rectangle of duration 1, at time instant $t=0.2$. Both values from the lumped circuit (dashed) and its compact model are reported.

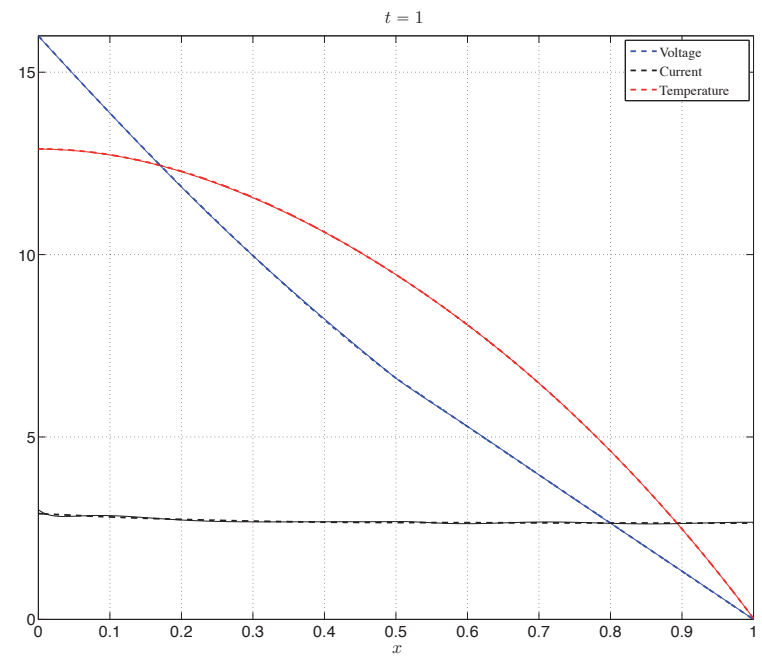

Fig. 2. Spacial distribution of voltage, current and temperature rise due to a current $I(t)$ constituted by a unit rectangle of duration 1 , at time instant $t=1$. Both values from the lumped circuit (dashed) and its compact model are reported.

[2] Yu Qingjian, J.M.L. Wang, E.S. Kuh, "Passive multipoint moment matching model order reduction algorithm on multiport distributed interconnect networks," IEEE Transactions on Circuits and Systems I: Fundamental Theory and Applications, Vol. 46 , No. 1, pp. 140$160,1999$.

[3] L. Codecasa, D. D’Amore, P. Maffezzoni, "Electro-thermal resonance in MOSFET devices," Electronics Letters, Vol. 37, No. 1, pp. 57-58, 2001.

[4] L. Codecasa, D. D'Amore, P. Maffezzoni, "Thermal networks for electro-thermal analysis of power devices," Microelectronics Journal, Vol. 32, No. 10-11, pp. 817-822, 2001.

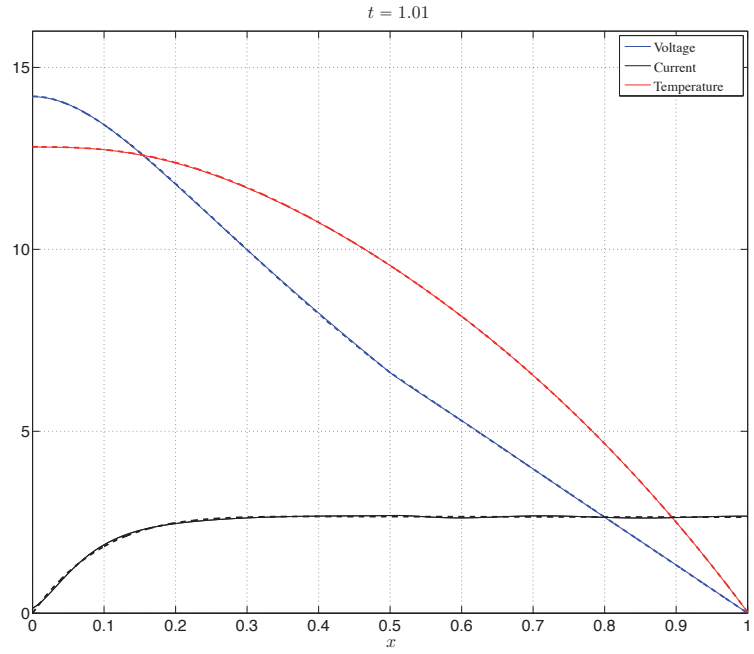

Fig. 3. Spacial distribution of voltage, current and temperature rise due to a current $I(t)$ constituted by a unit rectangle of duration 1, at time instant $t=1.01$. Both values from the lumped circuit (dashed) and its compact model are reported.

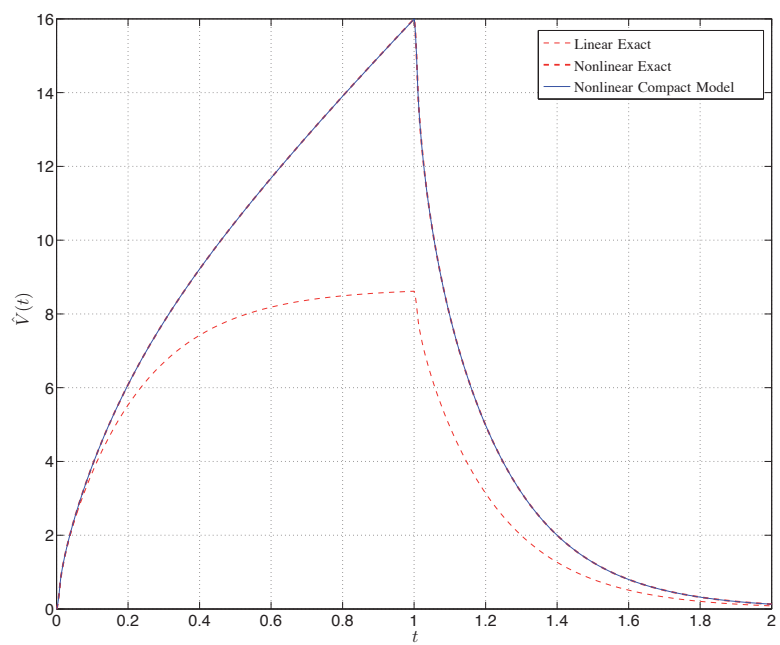

Fig. 4. Time distribution of the port voltage $\hat{V}(t)$ due to a current $I(t)$ constituted by a unit rectangle of duration 1 .

[5] L. Codecasa, D. D'Amore, P. Maffezzoni, "Modeling the thermal response of semiconductor devices through equivalent electrical networks," IEEE Transactions on Circuits and Systems I: Fundamental Theory and Applications, Vol. 49, No. 8, pp. 1187-1197, 2002.

[6] L. Codecasa, D. D'Amore, P. Maffezzoni, "An Arnoldi based thermal network reduction method for electro-thermal analysis," IEEE Transactions on Components and Packaging Technologies, Vol. 26, No. 1, pp. 186-192, 2003.

[7] L. Codecasa, D. D'Amore, P. Maffezzoni, "Compact modeling of electrical devices for electrothermal analysis," IEEE Transactions on Circuits and Systems I: Fundamental Theory and Applications, Vol. 50, No. 4, pp. 465-476, 2003. 


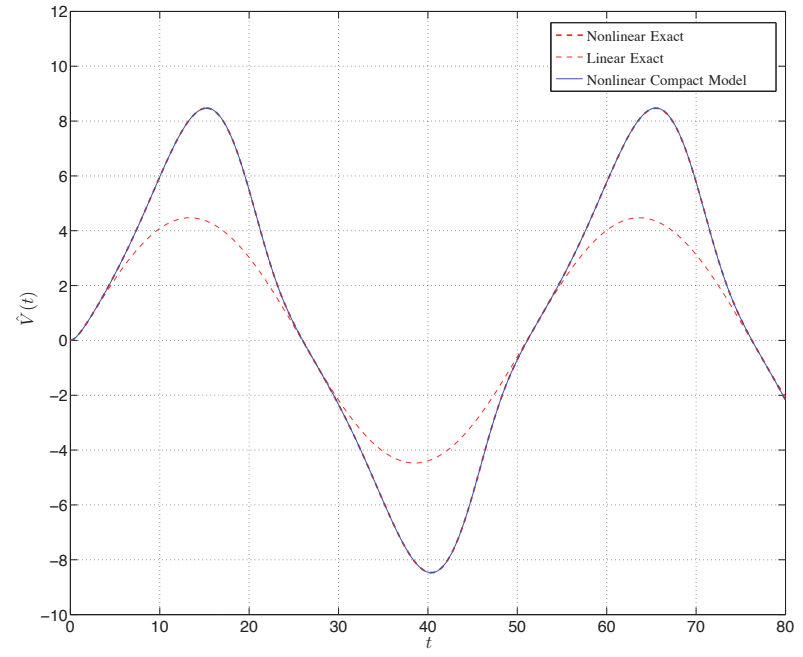

Fig. 5. Time distribution of the port voltage $\hat{V}(t)$ due to a sinusoidal current $I(t)$

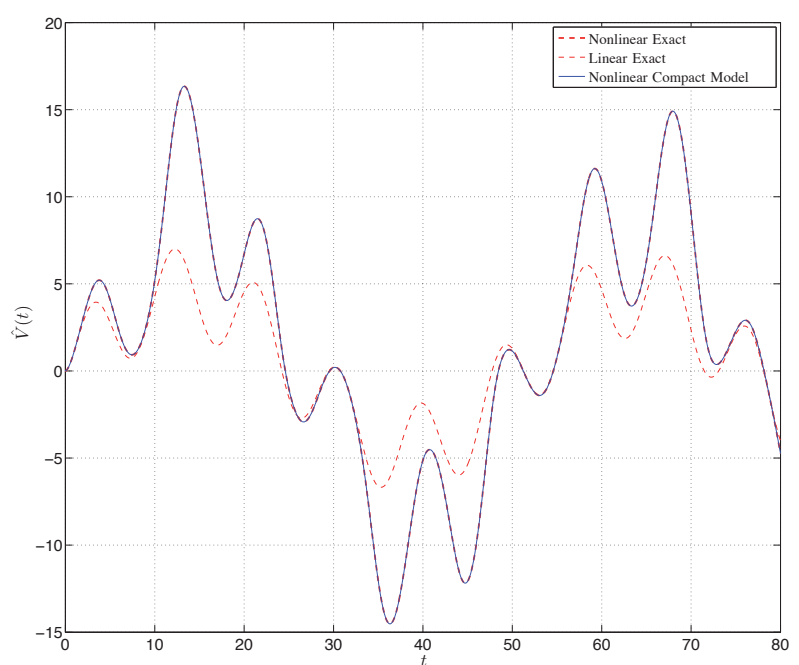

Fig. 6. Time distribution of the port voltage $\hat{V}(t)$ due to a current $I(t)$ composed of two sinusoidals.

[8] L. Codecasa, D. D'Amore, P. Maffezzoni, "Accurate electro-therma model of avalanching junctions subject to ESD currents," Electronics Letters, Vol. 39, No. 12, pp. 932-933, 2003.

[9] L. Codecasa, D. D'Amore, P. Maffezzoni, "Analytical multipoint moment matching reduction of distributed thermal networks," IEEE Transactions on Components and Packaging Technologies, Vol. 27, No. 1, pp. 87-95, 2004.

[10] L. Codecasa, D. D'Amore, P. Maffezzoni, "Compact thermal net works for modeling packages," IEEE Transactions on Components and Packaging Technologies, Vol. 27, No. 1, pp. 96-103, 2004.

[11] L. Codecasa, "Electro-thermal chaotic oscillations of paralleled bipolar transistors," Microelectronics Journal, Vol. 35, No. 10, pp. 859868,2004
[12] L. Codecasa, "Canonical forms of one-port passive distributed thermal networks," IEEE Transactions on Components and Packaging Technologies, Vol. 28, No. 1, pp. 5-13, 2005.

[13] L. Codecasa, "Thermal networks from heat wave equation," IEEE Transactions on Components and Packaging Technologies, Vol. 28, No. 1, pp. 14-22, 2005.

[14] L. Codecasa, D. D'Amore, P. Maffezzoni, "Multipoint moment matching reduction from port responses of dynamic thermal networks," IEEE Transactions on Components and Packaging Technologies, Vol. 28, No. 4, pp. 605-614, 2005.

[15] L. Codecasa, "A novel approach for generating boundary condition independent compact dynamic thermal networks of packages," IEEE Transactions on Components and Packaging Technologies, Vol. 28 , No. 4, pp. 593-604, 2005.

[16] L. Codecasa, D. D'Amore, P. Maffezzoni, "Physical interpretation and numerical approximation of structure functions of components and packages," 21st Annual IEEE Semiconductor Thermal Measurement and Management Symposium (SEMITHERM), pp. 146-153, 2005.

[17] L. Codecasa, D. D'Amore, P. Maffezzoni, "Multivariate moment matching for generating boundary condition independent compact dynamic thermal networks of packages," 21st Annual IEEE Semiconductor Thermal Measurement and Management Symposium (SEMITHERM), pp. 175-181, 2005.

[18] L. Codecasa, D. D'Amore, P. Maffezzoni, "Parametric compact models by directional moment matching," IEEE International Symposium on Circuits and Systems (ISCAS), pp. 1112-1114.

19] L. Codecasa, "Compact models of dynamic thermal networks with many heat sources," IEEE Transactions on Components and Packaging Technologies, Vol. 30, No. 4, pp. 653-659, 2007.

[20] L. Codecasa, "Structure function representation of multidirectional heat-flows," IEEE Transactions on Components and Packaging Technologies, Vol. 30, No. 4, pp. 643-652, 2007.

[21] P. Maffezzoni, L. Codecasa, D. D’Amore, M. Santomauro, "Semiimplicit integration method for the time-domain simulation of thermal responses," IEEE International Symposium on Circuits and Systems (ISCAS), pp. 816-819, 2008.

[22] L. Codecasa, D. D'Amore, P. Maffezzoni, "Compact thermal networks for conjugate heat transfer by moment matching," International Workshop on THERMal INvestigation of ICs and Systems (THERMINIC), pp. 52-57, 2008.

[23] L. Codecasa, D. D'Amore, P. Maffezzoni, "Triangulation method for structure functions of multi-directional heat-flows," International Workshop on THERMal INvestigation of ICs and Systems (THERMINIC), pp. 8-13, 2008.

24] L. Codecasa, D. D'Amore, P. Maffezzoni, "Multi-Physics Analysis of a Photovoltaic Panel with a Heat Recovery System ," International Workshop on THERMal INvestigation of ICs and Systems (THERMINIC), pp. 93-96, 2008.

[25] N. Spennagallo, L. Codecasa, D. D'Amore, P. Maffezzoni, "Evaluating the effects of temperature gradients and currents nonuniformity in on-chip interconnects," Microelectronics Journal, Vol. 40, No. 7 , pp 1154-1159, 2009.

[26] P. Maffezzoni, L. Codecasa, D. D'Amore, "Modeling and simulation of a hybrid photovoltaic module equipped with a heat-recovery system," IEEE Transactions on Industrial Electronics, Vol. 56, No. 11, pp. 4311-4318, 2009.

[27] L. Codecasa, L. Di Rienzo "Stochastic Thermal Modeling by Polynomial Chaos Expansion, International Workshop on THERMal INvestigation of ICs and Systems (THERMINIC), 2013.

[28] L. Codecasa, "Novel Approach to Compact Modeling for Nonlinear Thermal Conduction Problems", International Workshop on THERMal INvestigation of ICs and Systems (THERMINIC), 2013.

[29] W. J., Rugh, Nonlinear System Theory: The VolterraWiener Approach, Johns Hopkins University Press, Baltimore, 1981. 